\title{
Stabilized bordered block diagonal forms for parallel sparse solvers ${ }^{1,2}$
}

\author{
Iain S. Duff and Jennifer A. Scott
}

\begin{abstract}
We use the duality between a singly bordered and a doubly bordered block diagonal form to produce an efficient method of obtaining the stable factorization of a bordered system. This is particularly important in the exploitation of coarse-grained parallelism. We show how our partitioning and factorization scheme relates to domain decomposition partitioning but has greater control over stability. We propose using matrix stretching to generate a singly bordered form from a doubly bordered form. Matrix stretching is compared with two alternative methods for obtaining a singly bordered form and is shown to be efficient both in computation time and the quality of the resulting block structure.
\end{abstract}

Keywords: large sparse linear systems, unsymmetric matrices, ordering, partitioning, bordered block diagonal form, matrix stretching, parallel processing.

${ }^{1}$ Current reports available from "http://www.numerical.rl.ac.uk/reports/reports.html".

2 This work was supported by the EPSRC grants GR/R46641 and GR/S42170.

Computational Science and Engineering Department

Atlas Centre

Rutherford Appleton Laboratory

Oxon OX11 0QX

March 4, 2004. 


\section{Introduction}

Many large scale scientific and engineering computations require the solution of sparse linear systems of equations. In most applications, it is important to solve these systems as accurately and as rapidly as possible. In recent years, as the size of the problems of interest has increased, parallel algorithms and parallel computers have become very important for the efficient solution of these problems. Solving linear systems in parallel involves distributing the data and the computation among the processors. If the problem is well structured this can often be done in a straightforward way but the irregular structure of general large sparse unsymmetric systems can make the distribution of the data and computation difficult. A good ordering of the rows and columns of the matrix can significantly reduce the storage and computation time required to factorize it in parallel. The efficient computation of good orderings that can be used to obtain stable factorizations of unsymmetric problems is a major objective of this paper.

We are concerned with direct methods for solving linear systems of the form

$$
A x=b,
$$

where the $n \times n$ matrix $A=\left\{a_{i j}\right\}$ is large, sparse and unsymmetric. One possible approach to achieve coarse-grained parallelism is to preorder $A$ to bordered block diagonal form. The block diagonal form leads to subproblems that can be solved independently, leaving an interface problem corresponding to the border that links the subproblems. The interface problem must be solved to complete the solution of the original problem. For the method to work well in a parallel environment, the order of the interface problem should be small compared with $n$ so that there is little communication between the blocks on the diagonal and the interface problem and so that the cost of factorizing the interface problem is significantly less than that of factorizing the blocks on the diagonal. Recently, four solvers that implement direct algorithms based on this coarse-grained parallel approach have been developed for the mathematical software library HSL (HSL, 2002). These include the frontal solver HSL_MP43 (Scott, 2001) and HSL_MP48 (Duff and Scott, 2002) for sparse unstructured unsymmetric systems.

The matrix $A$ is said to be in doubly bordered block diagonal (DBBD) form if the rows and columns have been permuted to the form

$$
P A Q=A_{D B}=\left(\begin{array}{ccccc}
A_{11} & & & & C_{1} \\
& A_{22} & & & C_{2} \\
& & \ldots & & \cdot \\
& & & A_{N N} & C_{N} \\
R_{1} & R_{2} & \ldots & R_{N} & E
\end{array}\right),
$$

where the blocks $A_{l l}$ are $n_{l} \times n_{l}$ matrices and the border blocks $C_{l}$ and $R_{l}$ are $n_{l} \times p$ and $p \times n_{l}$ matrices, respectively, with $p \ll n_{l}$. This form is also sometimes referred to as an arrowhead form (see, for example, Ferris and Horn, 1998). For finite-element applications, the DBBD form corresponds to partitioning the underlying finite-element domain into non-overlapping subdomains; each block $A_{l l}, l=1,2, \ldots, N$, corresponds to the interior of a subdomain and the variables in the borders are those that lie on an interface between two or more subdomains. Each column of the column border $\left(C_{1}^{T}, C_{2}^{T}, \ldots, C_{N}^{T}, E^{T}\right)^{T}$ is 
called a coupling (or linking) column and each row of the row border $\left(R_{1}, R_{2}, \ldots, R_{N}, E\right)$ is a coupling (or linking) row. The aim is to permute $A$ to DBBD form in such a way that the number $p$ of coupling rows and columns is small, while at the same time ensuring a good balance between the sizes of the blocks $A_{l l}, l=1,2, \ldots, N$.

Having ordered $A$ into DBBD form, we can attempt to apply a linear solver to the blocks $A_{l l}$. However, there are potential problems in doing this. In particular, the $A_{l l}$ may be singular even if $A$ is nonsingular. It may be possible to use a direct solver that incorporates threshold-based partial pivoting for stability and moves rows and columns into the border as necessary, but the border size may grow too much. To avoid this problem, we propose using an alternative approach in which $A$ is ordered to singly bordered block diagonal (SBBD) form

$$
P A Q=A_{S B}=\left(\begin{array}{ccccc}
A_{11} & & & & C_{1} \\
& A_{22} & & & C_{2} \\
& & \ldots & & \cdot \\
& & & A_{N N} & C_{N}
\end{array}\right)
$$

where the blocks $A_{l l}$ are now rectangular $m_{l} \times n_{l}$ matrices with $m_{l} \geq n_{l}$, and the border blocks $C_{l}$ are of order $m_{l} \times p\left(p \ll n_{l}\right)$. Again, the objective is to permute $A$ into an SBBD form $A_{S B}$ so that the number of coupling columns is as small as possible while maintaining well balanced blocks $A_{l l}, l=1,2, \ldots, N$. Ideally the $A_{l l}$ should be well balanced in terms of factorization time, but at the very least we would plan that they have a similar dimension. With this form, a direct solver can be applied to factorize each of the rectangular blocks $A_{l l}$. We will show (see Section 2) that this in turn yields a stabilized DBBD form, that is, a DBBD form with nonsingular blocks on the diagonal that can be stably factorized without the need to delay pivots. In practice, parallel direct solvers such as HSL MP43 and HSL_MP48 do not form the stabilized DBBD form explicitly, but it can be used to provide a modified block Jacobi preconditioner for an iterative scheme on the whole system (Duff, Golub, Kwok and Scott 2004).

This paper is organised as follows. In Section 2, we look at how the SBBD form may be used with a direct solver to obtain a stabilized DBBD form. In Section 3, we discuss the reduction of unsymmetric sparse matrices to SBBD form and, in particular, we propose using a two-phase approach that is fast and yields a narrow border for a set of test problems arising from a range of practical applications that includes both highly unsymmetric problems and problems with a (nearly) symmetric sparsity structure. Our findings are summarised in Section 4.

All numerical experiments presented in this paper were performed on a single processor of a dual processor Compaq DS20 Alpha workstation, with 3.6 GBytes of RAM. The Fortran codes were compiled using the Compaq Fortran 90 compiler with the optimization flag -0; C codes were compiled using the Compaq cc compiler with the flag -04. 


\section{Stabilized DBBD forms}

If we are factorizing a block $A_{l l}$ of a doubly bordered block diagonal form (1.2) with threshold pivoting, then a potential pivot $a_{i j} \in A_{l l}$ must satisfy

$$
\left|a_{i j}\right| \leq u * \max \left\{\max _{k}\left|\left(A_{l l}\right)_{k j}\right|, \max _{k}\left|\left(R_{l}\right)_{k j}\right|\right\}
$$

where $u \in(0,1)$ is the threshold parameter. It is clear that large entries in the row border matrix $R_{l}$ can prevent potential pivots from being selected. Thus if we want to maintain the doubly bordered structure, we would court instability by failing to satisfy the threshold test. In order to maintain stability, we must move the rows and columns that cannot be eliminated to the borders thus changing the structure of the doubly bordered form. This is exactly the situation that we would encounter if we obtained such a DBBD form from a domain decomposition algorithm unless the matrix had special numerical properties, for example was positive definite. The increase in the border size could adversely affect the a priori data structures, will increase the size of the interface problem, increase the work for the factorization, and reduce the potential for parallelism. Furthermore, not being able to choose a full set of pivots from the blocks on the diagonal (in the extreme case it is possible that no pivots can be stably chosen) may cause problems with load balancing.

If, however, we are factorizing blocks $A_{l l}$ of a singly bordered block diagonal form (1.3) with threshold pivoting, then the potential pivot $a_{i j} \in A_{l l}$ must satisfy

$$
\left|a_{i j}\right| \leq u * \max _{k}\left\{\left|\left(A_{l l}\right)_{k j}\right|\right\}
$$

(again $u \in(0,1)$ is the threshold parameter) and so, if the matrix $A$ is nonsingular, there will always be a numerically satisfactory pivot in column $j$ of $A_{l l}$. It is thus always possible to factorize the submatrix $A_{l l}$ as the product of an $m_{l} \times n_{l}$ lower trapezoidal matrix $\bar{L}_{l}=\left(\begin{array}{c}L_{l} \\ \tilde{L}_{l}\end{array}\right)$ (where $L_{l}$ is an $n_{l} \times n_{l}$ lower triangular matrix) and an $n_{l} \times n_{l}$ upper triangular matrix, $U_{l}$.

We can look at the effect of this factorization on the SBBD matrix by considering the factorization of the augmented system $\left(\begin{array}{ll}A_{l l} & C_{l}\end{array}\right)$, which may be written in the form

$$
\left(\begin{array}{ll}
A_{l l} & C_{l}
\end{array}\right)=P_{l}\left(\begin{array}{ll}
L_{l} & \\
\tilde{L}_{l} & I
\end{array}\right)\left(\begin{array}{cc}
U_{l} & \tilde{U}_{l} \\
& S_{l}
\end{array}\right)\left(\begin{array}{cc}
Q_{l} & \\
& I
\end{array}\right),
$$

where $P_{l}$ and $Q_{l}$ are permutation matrices of order $m_{l} \times m_{l}$ and $n_{l} \times n_{l}$, respectively, and $S_{l}$ is an $\left(m_{l}-n_{l}\right) \times p_{l}$ local Schur complement matrix. We note that pivots cannot be chosen from the columns of $C_{l}$ because these columns have entries in at least one other border block $C_{j}(j \neq l)$. Expressing the factorization in this way enables us to identify a doubly bordered block diagonal form, where the coupling columns are as in the SBBD form and the coupling rows comprise the rows in the $\tilde{L}_{l}$ blocks. We observe that this DBBD form is rather special inasmuch as the set of rows in each border block $\tilde{L}_{l}$ is disjoint from the

rows in the other border blocks $\tilde{L}_{j}(j \neq l)$. This is part of the reason that the borders of this "stabilized" DBBD form are larger than for the DBBD form obtained directly using a graph partitioning tool such as the METIS package of Karypis and Kumar (1998) (see 


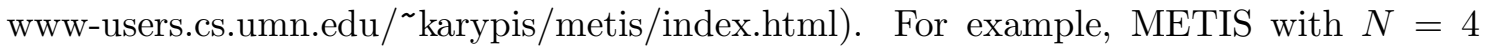
gives doubly bordered block diagonal forms with border sizes of 47 and 68 for problems bcircuit and nopoly, respectively (see Section 3.2 for details of our test problems). For the stabilized DBBD forms the corresponding border sizes are 94 and 136. However, the main point is that the DBBD form obtained via an SBBD form is stabilized in the sense that pivots can be stably chosen from within the blocks on the diagonal and no further alterations to the DBBD form will be necessary.

\section{Ordering to SBBD form}

The reduction of sparse unsymmetric matrices to SBBD form has been the subject of a number of recent papers (see, for example, Camarda and Stadtherr, 1998, Hu, Maguire and Blake, 2000, and Aykanat, Pinar and Çatalyürek, 2002 and the references therein). Hu et al. (2000) developed the MONET (Matrix Ordering for minimal NET-cut) algorithm for ordering chemical process engineering problems to SBBD form. MONET is based on applying a multilevel approach to the weighted row connectivity graph of $A$ (Mayoh, 1965). Given the original matrix, a series of matrices are generated, each coarser than the preceding one and obtained by merging rows with similiar sparsity patterns. The coarsest matrix is bisected. This bisection is prolonged to the finer matrices and refined using the Kernighan-Lin algorithm (Kernighan and Lin 1970). Partitioning into more than two blocks is achieved by recursive bisection. The net-cut is the number of columns that lie in the border, that is, the number of coupling columns. The algorithm aims to minimise the net-cut while ensuring good row balance (that is, each block $A_{l l}, l=1,2, \ldots, N$, has a similar number of rows).

HSL offers a Fortran 95 implementation of the MONET algorithm as routine HSL MC66. This code was used by Duff and Scott (2002) for preordering in their experiments with the parallel unsymmetric direct solvers HSL_MP43 and HSL_MP48. They found that, for highly unsymmetric problems, such as those that arise in chemical process engineering applications, HSL_MC66 produces high quality SBBD forms, that is, SBBD forms that are well balanced and have a narrow border. In particular, the numerical experiments reported by Duff and Scott showed that for up to 8 submatrices, the border typically represents less than $5 \%$ of the total number of columns. The narrow border and the balanced blocksize allows the parallel solvers to achieve good speedups when run on a modest number of processors (experiments using up to $N=16$ blocks on 16 processors were reported on). The main disadvantage of using the MONET algorithm is that it is relatively expensive in terms of CPU time. In general, the CPU cost of computing the singly bordered block form using HSL_MC66 was found to be significantly greater than the cost of the analyse phase of the direct solver applied to the blocks on the diagonal and, for some problems, it dominated the total solution time. Clearly, if a large number of matrices with the same sparsity pattern are to be factorized (such as when the linear solver is used within an iterative scheme for solving a nonlinear system), the ordering cost may be justified as it can be amortized over the repeated factorizations. But in some applications, only a single factorization is required and it may then be essential for the ordering to SBBD form to be performed rapidly so that it does not represent an unacceptable overhead. 
This is especially important if (as with HSL_MC66) the ordering is performed using a single processor.

The high cost of HSL_MC66 for large problems prompted Hu and Scott (2003) to look at developing alternative algorithms that avoid using the row graph of $A$. Their algorithms are based on computing either a vertex separator or a wide separator of the symmetrized matrix $A+A^{T}$. A graph partitioning tool such as METIS is used to partition the graph of $A+A^{T}$ and a vertex separator is extracted from the output. This is optionally widened to a wide separator and used to partition the matrix. Hu and Scott (2003) report on a number of variants of the separator approach. In this study, we use the method termed SEP_VS(ND). This employs the multilevel nested dissection routine METIS_NodeND; the METIS output is used to compute an SSBD form using both a vertex separator and a wide separator approach and the one that gives the narrowest border is selected.

Numerical experiments presented by $\mathrm{Hu}$ and Scott show that for $N \leq 8$ the number of coupling columns obtained using the separator methods is generally competitive with results for the MONET algorithm. Furthermore, employing the separator methods is significantly faster than using the MONET algorithm. This makes them useful alternatives, particularly if the required number of factorizations of matrices having the same sparsity pattern is small.

\subsection{Two-phase $A$ to $A_{S B}$ algorithm}

The separator algorithms of $\mathrm{Hu}$ and Scott and the MONET algorithm are one-phase schemes, that is, the SBBD form $A_{S B}$ is computed directly from $A$. An alternative approach that has been used for LP problems is a two-phase algorithm (Ferris and Horn, 1998, see also Aykanat et al., 2002). In the first phase, $A$ is ordered to DBBD form $A_{D B}$; then, in the second phase, row stretching is used to obtain an SBBD form $\hat{A}_{S B}$.

Stretching is a sparse matrix preprocessing technique that makes matrices sparser but, at the same time, larger. The idea was first introduced by Grcar (1990) who proposed both row and column stretching as an effective way of treating sparse matrices with dense rows or columns before factorization. The technique has since been used by a number of authors, including Alvarado (1997), Daydé, Décamps and Gould (1997), Ferris and Horn (1998), and Aykanat et al. (2002). We use row stretching to associate with $A_{D B}$ a larger square matrix $\hat{A}_{S B}$ in SBBD form. As an illustration, consider the $5 \times 52$-block DBBD matrix given by

$$
A_{D B}=\left(\begin{array}{ccccc}
\times & \times & & & \\
\times & \times & & & \\
& & \times & \times & \\
& & \times & \times & \times \\
\times & & \times & & \times
\end{array}\right) .
$$

The stretching process has two steps. In the first step, a rectangular matrix is constructed where the entries of the coupling rows (in this example, row 5 is the only coupling row) 
are split so that each new row has nonzero entries in only one non-border block:

$$
\left(\begin{array}{ccccc}
\times & \times & & & \\
\times & \times & & & \\
& & \times & \times & \\
& & \times & \times & \times \\
\times & & & & \times \\
& & \times & &
\end{array}\right)
$$

The second step produces a square matrix by appending new columns to ensure the stretched matrix is structurally nonsingular (provided $A_{D B}$ was structurally nonsingular). After reordering, the stretched matrix becomes:

$$
\hat{A}_{S B}=\left(\begin{array}{ccccccc}
\times & \times & & & \times & \\
\times & \times & & & & \\
\times & & & & \times & +\sigma \\
& & \times & \times & & \\
& & \times & \times & \times & \\
& & \times & & & -\sigma
\end{array}\right) .
$$

Here, $+\sigma$ and $-\sigma$ denote the added entries. More generally, suppose the row border $\left(R_{1}, R_{2}, \ldots, R_{N}, E\right)$ of the DBBD form $A_{D B}$ (see (1.2)) has $p$ rows and let row $i$ be the first row in the border. Assume row $i$ has nonzero entries in blocks $R_{i_{1}}, R_{i_{2}}, \ldots, R_{i_{k}}$ and $E$ $(k \geq 2)$. We start the stretching by removing the entries in blocks $R_{i_{2}}, \ldots, R_{i_{k}}$ from row $i$ and adding $k-1$ rows to $A_{D B}$ such that row $n+l$ contains the nonzero entries from row $i$ belonging to block $R_{i_{l+1}}, l=1, \ldots, k-1$. Next, we append $k-1$ columns to the border of $A_{D B}$ so that column $n+l$ has nonzero entries $\pm \sigma_{i_{l}}$ in rows $i$ and $n+l(l=1, \ldots, k-1)$. Finally, we permute the rows of the stretched matrix so that row $n+l$ is permuted to be a row of the block $A_{\hat{i} \hat{i}}$, where $\hat{i}=i_{l+1}$. We now have a DBBD form $\hat{A}_{D B}$ of order $(n+k-1) \times(n+k-1)$ with $p-1$ rows in its border. The process is repeated until finally the original DBBD has been stretched to a SBBD form $\hat{A}_{S B}$.

This technique of adding rows (and corresponding columns) to make the matrix sparser is also called row splitting (see, for example, Ferris and Horn (1998)). An analysis of the numerical properties of stretching is given by Grcar (1990). Assuming the original matrix $A$ is well scaled, the added entries $\pm \sigma_{i_{l}}$ should be chosen to ensure $\hat{A}_{S B}$ is also well scaled.

\subsection{One-phase and two-phase results for $A$ to SBBD form}

In this section, we present numerical results comparing the performance of the MONET algorithm, the separator algorithm SEP_VS(ND) of $\mathrm{Hu}$ and Scott, and the two-phase approach based on row splitting. Our test problems are listed in Table 3.1. For the coarse-grained parallel approach to be efficient, the test problems need to be reasonably large and so our selected examples are all of order at least 10,000. A † indicates that the problem is included in the University of Florida Sparse Matrix Collection (Davis, 1997). The remaining problems were supplied by Mark Stadtherr of the University of Notre Dame and Tony Garrett of AspenTech, UK. 


\begin{tabular}{|c|c|c|c|c|c|}
\hline Identifier & $n$ & $n z$ & $s(A)$ & $s(P A)$ & Description/Application area \\
\hline Matrix35640 & 35640 & 146880 & 0.0001 & 0.0427 & Chemical process engineering \\
\hline bayer $01^{\dagger}$ & 57735 & 277774 & 0.0002 & 0.0719 & Chemical process engineering \\
\hline icomp & 75724 & 338711 & 0.0010 & 0.0025 & Chemical process engineering \\
\hline Matrix32406 & 32406 & 1035989 & 0.0014 & 0.2643 & Chemical process engineering \\
\hline $\operatorname{lhr} 34 \mathrm{c}^{\dagger}$ & 35152 & 764014 & 0.0015 & 0.3294 & Chemical process engineering \\
\hline bayer0 $4^{\dagger}$ & 20545 & 159082 & 0.0016 & 0.0694 & Chemical process engineering \\
\hline $\operatorname{lh} r 71 \mathrm{c}^{\dagger}$ & 70304 & 1528092 & 0.0016 & 0.3541 & Chemical process engineering \\
\hline poli_large ${ }^{\dagger}$ & 15575 & 33074 & 0.0035 & 0.0035 & Account of capital links \\
\hline $4 \mathrm{cols}$ & 11770 & 43668 & 0.0159 & 0.0419 & Chemical process engineering \\
\hline $10 \mathrm{cols}$ & 29496 & 109588 & 0.0167 & 0.0471 & Chemical process engineering \\
\hline onetone $2^{\dagger}$ & 36057 & 227628 & 0.1129 & 0.3600 & Harmonic balance method \\
\hline ethylene-1 & 10673 & 80904 & 0.2973 & 0.2441 & Chemical process engineering \\
\hline ethylene-2 & 10353 & 78004 & 0.3020 & 0.2487 & Chemical process engineering \\
\hline circuit_3 $3^{\dagger}$ & 12127 & 48137 & 0.7701 & 0.4093 & Circuit simulation \\
\hline circuit_4 $4^{\dagger}$ & 80209 & 79566 & 0.8292 & 0.7894 & Circuit simulation \\
\hline Zhao $2^{\dagger}$ & 33861 & 166453 & 0.9225 & & Electromagnetics \\
\hline scircuit $^{\dagger}$ & 170998 & 958936 & 0.9999 & & Circuit simulation \\
\hline hcircuit $^{\dagger}$ & 105676 & 513072 & 0.9999 & & Circuit simulation \\
\hline bcircuit $^{\dagger}$ & 68902 & 375558 & 1.0000 & & Circuit simulation \\
\hline garon $2^{\dagger}$ & 13535 & 390607 & 1.0000 & & 2D Navier Stokes \\
\hline pesa $^{\dagger}$ & 11738 & 79566 & 1.0000 & & Unknown \\
\hline venkat $50^{\dagger}$ & 62424 & 1717792 & 1.0000 & & 2D Euler \\
\hline nopoly ${ }^{\dagger}$ & 10774 & 70842 & 1.0000 & & Unknown \\
\hline
\end{tabular}

Table 3.1: Test problems. $n, n z$ denote the order of $A$ and the number of matrix entries, respectively. $s(A)$ denotes the symmetry index and $s(P A)$ is the symmetry index after ordering with MC21. Problems marked $\dagger$ are available from the University of Florida Sparse Matrix Collection. 
The symmetry index $s(A)$ of a matrix $A$ is defined to be the number of matched nonzero off-diagonal entries (that is, the number of nonzero entries $a_{i j}, i \neq j$, for which $a_{j i}$ is also nonzero) divided by the total number $n z$ of off-diagonal nonzero entries. Small values of $s(A)$ indicate the matrix is far from symmetric while values close to 1 indicate an almost symmetric sparsity pattern. In Table 3.1, the test matrices are listed in order of increasing symmetry index. A number of the matrices are highly unsymmetric and have a large number of zero diagonal entries. For such problems, matching orderings generally increase the symmetry index of the resulting reordered matrix (see, for example, Duff and Koster, 1999). The HSL routine MC21 uses a relatively simple algorithm to compute a matching that corresponds to a row permutation of $A$ that puts nonzeros entries onto the diagonal, without considering the numerical values (for details see Duff, 1981a, 1981b). The numbers in column 5 of Table 3.1 are the symmetry indices after applying MC21 to the problems with $s(A) \leq 0.9$. We see that, in general, $s(A)$ is increased by applying MC21 and for some of the highly unsymmetric problems (including Matrix32406, 1hr34c and $1 \mathrm{hr} 71 \mathrm{c}$ ) the increase is substantial. There is no guarantee that MC21 will increase $s(A)$ and, for a number of the problems with a relatively large symmetry index, we find that $s(P A)$ (where $P$ is the permutation returned by MC21) is smaller than $s(A)$. These problems have either no zero diagonal entries or a very small number of zeros on the diagonal (for example, circuit_4 has only 21 zeros on the diagonal) and in such cases changes to $s(A)$ resulting from using MC21 are largely due to the tie-breaking within MC21.

For the test problems in the top half of the table with an unsymmetric sparsity pattern up to and including problem onetone2, we let $B=P A$ and for the remaining problems we set $B=A$. Hu and Scott (2003) report that if $A$ has a small symmetry index the vertex separator methods yield narrower borders when applied to $B$ instead of $A$; for highly unsymmetric problems with many zeros on the diagonal, the reduction in border size by preordering with MC21 can be substantial. Thus, in the remainder of this section, the separator methods are applied to $B$ (and, when used, the time for MC21 is included in the time needed to compute the SBBD form). Note that the MONET algorithm is applied to $A$ because it is designed particularly for highly unsymmetric problems; experiments have shown that applying it to $B$ generally results in wider borders.

For the stretching methods, in the first phase we must permute $A$ to DBBD form $A_{D B}$. For symmetrically structured matrices this is done by computing a vertex separator $V_{s}$ of the adjacency graph of $A$; the coupling rows and columns of $A_{D B}$ correspond to the vertices belonging to $V_{s}$. In our experiments we use the METIS package and, in particular, the multilevel nested dissection routine METIS NodeND. We note that minor modifications were needed in order to extract the vertex separator information. The output from the modified METIS_NodeND is an array part of length $n$. If part (i) $=0$ then vertex $i \in V_{s}$; otherwise, if part ( $i)=\mathrm{k}(1 \leq k \leq N)$, vertex $i$ belongs to the $k$ th block of $A_{D B}$.

For problems with an unsymmetric sparsity pattern we report the results of applying METIS_NodeND to the graph of the matrix $A+A^{T}$ and to the bipartite graph of $A$. When applied to $A+A^{T}$, if vertex $i \in V_{s}$ then the $i$ th row and column of $A$ are moved to the row and column borders, respectively.

The bipartite graph of $A$ consists of two distinct sets $\mathcal{R}$ and $\mathcal{C}$ of $n$ vertices each and undirected edges joining the vertices in $\mathcal{R}$ with those in $\mathcal{C}$. The set $\mathcal{R}$ is associated with the rows of $A$ and the set $\mathcal{C}$ with the columns. There is an edge $\left(r_{i}, c_{j}\right)$ if and only if 
$r_{i} \in \mathcal{R}, c_{j} \in \mathcal{C}$ and $a_{i j}$ is nonzero. It is straightforward to show that the bipartite graph is equivalent to the adjacency graph of the $2 n \times 2 n$ matrix

$$
\left(\begin{array}{cc}
0 & A \\
A^{T} & 0
\end{array}\right) .
$$

METIS_NodeND is applied to this graph. If vertex $i \leq n$ belongs to $V_{s}$ then row $i$ of $A$ is permuted to the row border, while if $i>n$ belongs to $V_{s}$, column $j=i-n$ is permuted to the column border.

Once $A$ has been permuted to a DBBD form $A_{D B}$, stretching is used to obtain an SBBD form $\hat{A}_{S B}$ of order $\hat{n} \times \hat{n}$. We are concerned that the difference between $\hat{n}$ and $n$ (the size of the original matrix $A$ ) should be small relative to $n$. This is important because we now have a larger (stretched) linear system of the form

$$
\hat{A}_{S B} \hat{x}=\hat{b}
$$

to solve in place of the original system (1.1). In Table 3.2, $n$ is compared with $\hat{n}$ for each of our test examples with $N=8$ blocks. The size of $\hat{n}$ depends on the method used to permute $A$ to DBBD form. The results of applying METIS Nodend to $A+A^{T}\left(\hat{n}_{1}\right)$, to the graph of $B+B^{T}\left(\hat{n}_{2}\right)$ and to the bipartite graph $\left(\hat{n}_{3}\right)$ are given in Table 3.2. A blank entry in the $\hat{n}_{2}$ column indicates the problem was not preordered using MC21 (that is, $B=A$ ), and there is a blank in the $\hat{n}_{3}$ column for the symmetric matrices because the bipartite graph is not used for these problems. For the problems with a symmetric pattern (problems bcircuit onwards), the results are for applying METIS to $A+A^{T}=A$. The figures in parentheses are the percentage increases in the order of the system. Comparing the $\hat{n}_{1}$ and $\hat{n}_{2}$ columns, we see that for the highly unsymmetric problems the number of rows and columns added during the stretching process is significantly reduced by preordering using MC21. If we compare the use of $B+B^{T}$ with that of the bipartite graph, we note that, in general, the former results in a slightly smaller stretched matrix. However, for each problem in the test set and $N=8$, both approaches lead to an increase in the matrix size of less than 6 percent. Further results for a subset of our test problems run with $N=4$ and $N=16$ are given in Table 3.3. In each case, METIS is applied to $B+B^{T}$ (with $B=A$ for the matrices below problem onetone2). We see that as $N$ increases so too does the order $\hat{n}$ of the stretched matrix but the increases are generally modest. In particular, with $N=16$ the increase in matrix size is less than 10 per cent for all our test problems (including those not reported on in Table 3.3) and for most problems the increase is less than 3 per cent. These results are encouraging since such a small increase is unlikely to add a significant overhead to the time needed to solve the linear system.

We now compare the border sizes of the two-phase approach with those obtained using the HSL_MC66 implementation of the MONET algorithm and the separator method SEP_VS(ND) of $\mathrm{Hu}$ and Scott. Default setting are used for all HSL_MC66 control parameters. For the two-phase approach we apply METIS to the graph of $B+B^{T}$ and, for matrices with an unsymmetric sparsity pattern, to the bipartite graph. Results are given in Table 3.4 for $N=8$. We highlight in bold the narrowest borders and those that are within 5 percent of the best. The results show that the two-phase approach works well, both for the problems with an unsymmetric structure and those that are symmetric. In particular, 


\begin{tabular}{|l||rrrrrrr|}
\hline \multicolumn{1}{|c||}{ Identifier } & \multicolumn{1}{c}{$n$} & \multicolumn{2}{c}{$\hat{n}_{1}$} & \multicolumn{3}{c|}{$\hat{n}_{2}$} & \multicolumn{3}{c|}{$\hat{n}_{3}$} \\
\hline Matrix35640 & 35640 & 50361 & $(41.3)$ & 35981 & $(0.96)$ & 36605 & $(2.72)$ \\
bayer01 & 57735 & 61940 & $(7.28)$ & 57916 & $(0.31)$ & 57963 & $(0.39)$ \\
icomp & 75724 & 75907 & $(0.24)$ & 75864 & $(0.18)$ & 75868 & $(0.19)$ \\
Matrix32406 & 32406 & 35823 & $(10.5)$ & 33212 & $(2.48)$ & 33241 & $(2.57)$ \\
lhr34c & 35152 & 40662 & $(15.7)$ & 35558 & $(1.15)$ & 35670 & $(1.47)$ \\
bayer04 & 20545 & 23159 & $(12.7)$ & 20770 & $(1.09)$ & 20846 & $(1.46)$ \\
lhr71c & 70304 & 74296 & $(5.68)$ & 70764 & $(0.65)$ & 70690 & $(0.55)$ \\
poli_large & 15575 & 15927 & $(2.26)$ & 15927 & $(2.26)$ & 16059 & $(3.10)$ \\
4cols & 11770 & 11857 & $(7.39)$ & 11841 & $(0.60)$ & 11865 & $(0.81)$ \\
10cols & 29496 & 29622 & $(0.43)$ & 29602 & $(0.36)$ & 29621 & $(0.42)$ \\
onetone2 & 36057 & 36377 & $(0.89)$ & 36680 & $(1.72)$ & 36458 & $(1.11)$ \\
ethylene-1 & 10673 & 10722 & $(0.46)$ & & & 10730 & $(0.53)$ \\
ethylene-2 & 10353 & 10435 & $(0.79)$ & & & 10437 & $(0.81)$ \\
circuit_3 & 12127 & 12197 & $(0.33)$ & & & 12212 & $(0.70)$ \\
circuit_4 & 80209 & 81131 & $(1.15)$ & & & 80924 & $(0.89)$ \\
Zhao2 & 33861 & 35696 & $(5.42)$ & & & 35506 & $(4.85)$ \\
scircuit & 170998 & 171327 & $(0.19)$ & & & 171293 & $(0.17)$ \\
hcircuit & 105676 & 105900 & $(0.21)$ & & & 105937 & $(0.25)$ \\
bcircuit & 68902 & 69099 & $(0.26)$ & & & & \\
garon2 & 13535 & 14302 & $(5.67)$ & & & & \\
pesa & 11738 & 11934 & $(1.67)$ & & & & \\
venkat50 & 62424 & 63544 & $(1.79)$ & & & & \\
nopoly & 10774 & 10925 & $(1.40)$ & & & & \\
\hline
\end{tabular}

Table 3.2: The order of the stretched matrix $\hat{A}_{S B}(N=8)$. The figures in parentheses are the percentage increases in the order of the system.

\begin{tabular}{|l||ccccccc|}
\hline \multicolumn{1}{|c||}{ Identifier } & $n$ & \multicolumn{7}{c|}{$N=4$} & \multicolumn{2}{c|}{$N=8$} & \multicolumn{2}{c|}{$N=16$} \\
\hline Matrix35640 & 35640 & 35811 & $(0.48)$ & 35981 & $(0.96)$ & 36692 & $(2.95)$ \\
bayer01 & 57735 & 57829 & $(0.16)$ & 57916 & $(0.31)$ & 58022 & $(0.50)$ \\
lhr71c & 70304 & 70411 & $(0.15)$ & 70764 & $(0.65)$ & 71129 & $(1.17)$ \\
10cols & 29496 & 29534 & $(0.13)$ & 29602 & $(0.36)$ & 29688 & $(0.65)$ \\
onetone2 & 36057 & 36313 & $(0.73)$ & 36680 & $(1.72)$ & 36940 & $(2.45)$ \\
circuit_4 & 80209 & 80677 & $(0.58)$ & 81131 & $(1.15)$ & 81877 & $(2.08)$ \\
Zhao2 & 33861 & 34761 & $(2.66)$ & 35696 & $(5.42)$ & 37153 & $(9.72)$ \\
venkat50 & 62424 & 63096 & $(1.08)$ & 63544 & $(1.79)$ & 64356 & $(3.09)$ \\
nopoly & 10774 & 10842 & $(0.63)$ & 10925 & $(1.40)$ & 11011 & $(2.20)$ \\
\hline
\end{tabular}

Table 3.3: The order of the stretched matrix $\hat{A}_{S B}$ for different $N$. The figures in parentheses are the percentage increases in the order of the system. 


\begin{tabular}{|l||c|rrrr|}
\hline \multicolumn{1}{|c||}{ Identifier } & $n$ & SEP_VS(ND) & HSL_MC66 & \multicolumn{2}{c|}{ Two-phase } \\
& & & & $B+B^{T}$ & Bipartite \\
\hline Matrix35640 & 35640 & 1599 & $\mathbf{1 3 6 7}$ & 1514 & 1476 \\
bayer01 & 57735 & 458 & $\mathbf{2 5 4}$ & 431 & 385 \\
icomp & 75724 & 332 & $\mathbf{2 2 9}$ & $\mathbf{2 3 9}$ & 409 \\
Matrix32406 & 32406 & 1756 & 3514 & 1886 & $\mathbf{1 4 6 9}$ \\
lhr34c & 35152 & 941 & $\mathbf{7 9 2}$ & 1062 & $\mathbf{7 6 7}$ \\
bayer04 & 20545 & 404 & 542 & 547 & $\mathbf{3 6 7}$ \\
lhr71c & 70304 & 883 & 990 & 1163 & $\mathbf{8 3 2}$ \\
poli_large & 15575 & 2200 & 713 & $\mathbf{4 7 3}$ & $\mathbf{4 9 2}$ \\
4cols & 11770 & 263 & 233 & $\mathbf{2 0 4}$ & 268 \\
10cols & 29496 & 313 & $\mathbf{2 7 9}$ & $\mathbf{2 6 3}$ & 361 \\
onetone2 & 36057 & 1596 & 1745 & 1812 & $\mathbf{1 3 1 0}$ \\
ethylene-1 & 10673 & 190 & 217 & 215 & $\mathbf{1 6 8}$ \\
ethylene-2 & 10353 & 201 & 217 & $\mathbf{9 5}$ & 191 \\
circuit_3 & 12127 & 2015 & 1920 & $\mathbf{1 1 4}$ & 143 \\
circuit_4 & 80209 & 19305 & 16417 & 1107 & $\mathbf{9 6 2}$ \\
Zhao2 & 33861 & 3132 & $\mathbf{2 7 7 3}$ & 3209 & 3036 \\
scircuit & 170998 & 1274 & 4353 & $\mathbf{5 8 1}$ & 649 \\
hcircuit & 105676 & 2350 & 2138 & 370 & $\mathbf{1 5 4}$ \\
bcircuit & 68902 & 495 & 951 & $\mathbf{3 9 4}$ & \\
garon2 & 13535 & 2043 & 2308 & $\mathbf{1 5 1 6}$ & \\
pesa & 11738 & 469 & 446 & $\mathbf{3 9 1}$ & \\
venkat50 & 62424 & 2492 & 2536 & $\mathbf{2 2 3 2}$ & \\
nopoly & 10774 & 310 & 337 & $\mathbf{3 0 2}$ & \\
\hline
\end{tabular}

Table 3.4: The size of the border in the 8-block SBBD form computed using the SEP_VS(ND) method, HSL_MC66, and the two-phase approach. 
for the (nearly) symmetric problems it consistently outperforms the SEP_VS(ND) method, and there are only a small number of the highly unsymmetric problems for which HSL MC66 produces significantly smaller borders. For the circuit simulation problems, the two-phase approach succeeds in producing much narrower borders than the one-phase algorithms. For problem circuit_4, both SEP_VS(ND) and HSL_MC66 give borders that are too wide for the coarse-grained parallel approach to work well; the solution time will be dominated by the time needed to solve the interface problem.

Comparing the two versions of the two-phase approach, using the bipartite graph for the unsymmetric problems seems to produce slightly narrower borders than using the graph of $B+B^{T}$. However, the reordering times given in Table 3.5 show that using the bipartite graph is generally the more expensive approach. This is because it involves applying METIS to a graph with $2 n$ vertices while the graph of $B+B^{T}$ has only $n$ vertices. The difference in CPU times is significantly less for the unsymmetric problems that are preordered for the $B+B^{T}$ approach using MC21 since employing MC21 incurs an overhead. There is little difference between the SEP_VS(ND) and two-phase $B+B^{T}$ times and both

\begin{tabular}{|l||crrr|}
\hline \multicolumn{1}{|c||}{ Identifier } & SEP_VS(ND) & HSL_MC66 & \multicolumn{2}{c|}{ Two-phase } \\
& & & $B+B^{T}$ & Bipartite \\
\hline Matrix35640 & 1.57 & 6.93 & 1.54 & 2.02 \\
bayer01 & 2.06 & 6.33 & 2.25 & 2.19 \\
icomp & 1.64 & 5.71 & 1.67 & 2.82 \\
Matrix32406 & 5.27 & 324 & 5.45 & 9.48 \\
lhr34c & 3.12 & 9.64 & 3.30 & 3.47 \\
bayer04 & 0.85 & 3.70 & 0.86 & 1.09 \\
lhr71c & 7.58 & 19.3 & 7.51 & 7.16 \\
poli_large & 0.22 & 0.21 & 0.23 & 0.30 \\
4cols & 0.66 & 0.91 & 0.66 & 0.85 \\
10cols & 2.14 & 2.45 & 2.12 & 2.96 \\
onetone2 & 1.35 & 8.12 & 1.51 & 1.34 \\
ethylene-1 & 0.25 & 2.59 & 0.25 & 0.38 \\
ethylene-2 & 0.24 & 3.07 & 0.21 & 0.35 \\
circuit_3 & 0.20 & 51.6 & 0.20 & 0.35 \\
circuit_4 & 3.29 & 2121 & 3.74 & 6.46 \\
Zhao2 & 0.65 & 2.44 & 0.65 & 1.61 \\
scircuit & 2.83 & 54.7 & 2.70 & 7.78 \\
hcircuit & 1.23 & 16.1 & 1.19 & 2.91 \\
bcircuit & 0.95 & 5.34 & 0.97 & \\
garon2 & 0.27 & 2.35 & 0.34 & \\
pesa & 0.14 & 0.75 & 0.14 & \\
venkat50 & 0.82 & 6.14 & 1.26 & \\
nopoly & 0.12 & 0.68 & 0.12 & \\
\hline
\end{tabular}

Table 3.5: The CPU time (in seconds) to compute the 8-block SBBD form using the SEP_VS(ND) method, HSL_MC66, and the two-phase approach.

are significantly faster than HSL_MC66.

Finally, we consider the row imbalance. We define the row imbalance to be the difference between the maximum submatrix row dimension and the average submatrix row dimension, divided by the average submatrix row dimension, expressed as a percentage. 
That is, if $m_{l}$ is the row dimension of $A_{l l}$, then

$$
\text { row imbalance }=\frac{\max \left\{m_{l}\right\}-n / N}{n / N} \times 100 .
$$

For our stretching approach, the row imbalance can increase significantly with $N$. For example, for problem bayer04 the row balance is 4.2 per cent for $N=4,8.8$ per cent for $N=8$ and 13.3 per cent for $N=16$. For HSL $\_$MC66, the row imbalance increases more slowly with $N$ and for our test examples, the row imbalance was less than 5 per cent for $N \leq 16$. We remark that HSL_MC66 has a parameter that may be set by the user to control the size of the row imbalance and so, if the user is willing to allow a greater imbalance, it may be possible to obtain a narrower border. We also note that the real metrics for load balancing should be the amount of work and time required by the factorization and this is not necessarily proportional to the submatrix row dimension.

We conclude that the two-phase approach is very successful at efficiently computing SBBD forms with a narrow border. The method replaces the original linear system by a larger one, but in our tests the increase in the order of the linear system was modest. However, we may need to investigate in the future whether it is possible to improve the row imbalance as the number of blocks is increased.

\section{Concluding remarks}

We have shown a duality between doubly and singly bordered block diagonal forms and have used this, on the one hand, in an efficient algorithm to produce an SBBD form and, on the other hand, have used a stable factorization of the SBBD form to define a stabilized DBBD form.

The stabilized form can either be used to effect a direct solution of the system of equations, see for example Duff and Scott (2002), or as the basis for a block iterative technique, either using a Schur complement approach or a variant of block Jacobi (Duff et al. 2004).

We have also shown how matrix stretching can be used to generate a singly bordered form from a doubly bordered one. Numerical results have been presented for this approach and compared with using the MONET algorithm (HSL_MC66) and with methods based on vertex separators. The results show that matrix stretching is significantly faster than MONET and leads to only a modest increase in the order of the matrix. Moreover, matrix stretching produces border sizes that are competitive with MONET for highly unsymmetric problems and are significantly narrower for problems with a (nearly) symmetric sparsity pattern.

\section{Acknowledgements}

We are grateful to Yifan $\mathrm{Hu}$ of Wolfram Research for modifying METIS_NodeND to output vertex separator information and for commenting on a draft of this report. We would also like to thank our colleague John Reid for helpful comments. 


\section{References}

F.L. Alvarado. Matrix enlarging methods and their application. BIT, 37, 473-505, 1997.

C. Aykanat, A. Pinar, and Ü.V. Çatalyürek. Permuting sparse rectangular matrices into singly-bordered block-diagonal form for parallel solution of LP problems. Technical Report BU-CE-0203, Computer Engineering Department, Bilkent Univeristy, 2002.

K.V. Camarda and M.A. Stadtherr. Frontal solvers for process engineering: local row ordering strategies. Computers in Chemical Engineering, 22, 333-341, 1998.

T. Davis. University of Florida Sparse Matrix Collection. NA Digest, 97(23), 1997. Full details from www.cise.ufl.edu/ davis/sparse/.

M.J. Daydé, J.P. Décamps, and N.I.M Gould. Solution of unassembled linear systems using block stretching: preliminary experiments. Technical Report TR/APO/97/3, ENSEEIHT-IRIT, Toulouse, 1997.

I.S. Duff. Algorithm 575, permutations for a zero-free diagonal. ACM Trans. Mathematical Software, 7, 387-390, 1981a.

I.S. Duff. On algorithms for obtaining a maximum transversal. ACM Trans. Mathematical Software, 7, 315-330, $1981 b$.

I.S. Duff and J. Koster. The design and use of algorithms for permuting large entries to the diagonal of sparse matrices. SIAM J. Matrix Analysis and Applications, 20, 889-901, 1999 .

I.S. Duff and J.A. Scott. A parallel direct solver for large sparse highly unsymmetric linear systems. Technical Report RAL-TR-2002-033, Rutherford Appleton Laboratory, 2002. ACM Trans. Mathematical Software, to appear, 2004.

I.S Duff, G. Golub, F. Kwok, and J.A. Scott. Combining direct and iterative methods to solve partitioned linear systems. Technical Report, to appear, Rutherford Appleton Laboratory, 2004. Presented by Kwok at SIAM Meeting on Parallel Processing, San Francisco, February 2004.

M.C. Ferris and J.D. Horn. Partitioning mathematical programs for parallel solution. Mathematical Programming, 80, 35-62, 1998.

J.F. Grcar. Matrix stretching for linear equations. Technical Report SAND90-8723, Sandia National Laboratories, 1990.

HSL. A collection of Fortran codes for large-scale scientific computation, 2002. See http://hsl.rl.ac.uk/.

Y.F. Hu and J.A. Scott. Ordering techniques for singly bordered block diagonal forms for unsymmetric parallel sparse direct solvers. Technical Report RAL-TR-2003-020, Rutherford Appleton Laboratory, 2003. 
Y.F. Hu, K.C.F. Maguire, and R.J. Blake. A multilevel unsymmetric matrix ordering for parallel process simulation. Computers in Chemical Engineering, 23, 1631-1647, 2000 .

G. Karypis and V. Kumar. METIS: A software package for partitioning unstructured graphs, partitioning meshes and computing fill-reducing orderings of sparse matrices - version 4.0, 1998.

B.W. Kernighan and S. Lin. An efficient heuristic procedure for partitioning graphs. Bell Systems Tech. J., 49, 291-308, 1970.

B.H. Mayoh. A graph technique for inverting certain matrices. Mathematics of Computation, 19, 644-646, 1965.

J.A. Scott. Two-stage ordering for unsymmetric parallel row-by-row frontal solvers. Computers in Chemical Engineering, 25, 323-332, 2001. 